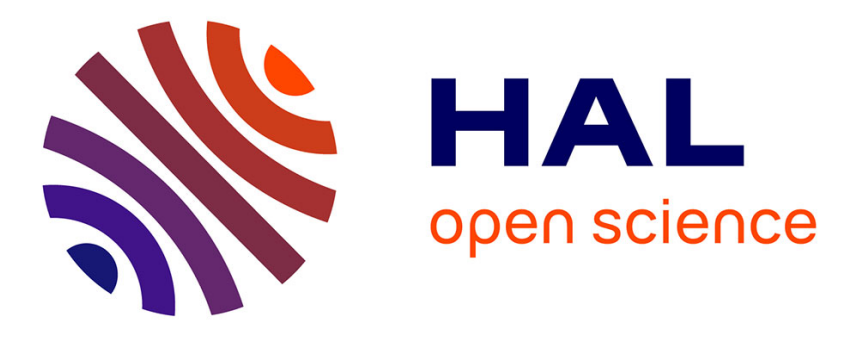

\title{
Polychromatic guide star: feasibility study
}

\author{
Renaud Foy, Jean-Paul Pique, Alain D. Petit, Patrick Chevrou, Vincent \\ Michau, Pascal Prigent, Gilbert Grynberg, Arnold Migus, Nancy Ageorges, \\ Veronique Bellanger, et al.
}

\section{- To cite this version:}

Renaud Foy, Jean-Paul Pique, Alain D. Petit, Patrick Chevrou, Vincent Michau, et al.. Polychromatic guide star: feasibility study. Proceedings of SPIE, the International Society for Optical Engineering, 2000, High-Power Laser Ablation III, Claude R. Phipps; Ed. Published, 4065, pp.312 - 323. hal01118342

\section{HAL Id: hal-01118342 \\ https://hal.science/hal-01118342}

Submitted on 5 Mar 2015

HAL is a multi-disciplinary open access archive for the deposit and dissemination of scientific research documents, whether they are published or not. The documents may come from teaching and research institutions in France or abroad, or from public or private research centers.
L'archive ouverte pluridisciplinaire HAL, est destinée au dépôt et à la diffusion de documents scientifiques de niveau recherche, publiés ou non, émanant des établissements d'enseignement et de recherche français ou étrangers, des laboratoires publics ou privés. 


\title{
Polychromatic guide star: Feasibility study.
}

\author{
Renaud Foy ${ }^{1}$, Jean-Paul Pique ${ }^{2}$, Alain Petit ${ }^{3}$, Patrick Chevrou ${ }^{4}$, \\ Vincent Michau ${ }^{5}$, Pascale Prigent ${ }^{6}$, Gilbert Grynberg ${ }^{7}$, Arnold Migus ${ }^{8}$, \\ Nancy Ageorges ${ }^{9}$, Véronique Bellanger ${ }^{2}$, François Biraben ${ }^{7}$, Ruy Deron ${ }^{5}$, Hayden Fews ${ }^{9}$, \\ Françoise Foy ${ }^{1}$, Claudia Högemann ${ }^{1}$, Pascal Jagourel ${ }^{6}$, Markus Laubscher ${ }^{2}$, \\ Daniel Müller ${ }^{2}$, Céline d'Orgeville ${ }^{2}$, Olivier Peillet ${ }^{4}$, Mike Redfern $^{9}$, Matthias Schöck ${ }^{1}$, \\ Patricia Segonds $^{2}$, Richard Soden ${ }^{2,1}$, Michel Tallon ${ }^{1}$, \\ Isabelle Tallon-Bosc ${ }^{1}$, Éric Thiébaut ${ }^{1}$, Andrë̈ Tokovinin ${ }^{1}$, Jérôme Vaillant ${ }^{1}$, Jean-Marc Weulersse ${ }^{3}$ \\ ${ }^{1}$ Centre de Recherches Astronomiques de Lyon (CRAL), \\ Observatoire de Lyon, 69561 Saint Genis Laval Cedex, France \\ ${ }^{2}$ Laboratoire de Spectrométrie Physique (LSP: UMR CNRS-UJF 5588), \\ Université Joseph Fourier de Grenoble, 38402 Saint Martin d'Hères Cedex, France \\ ${ }^{3}$ Commissariat à l'Énergie Atomique (CEA/DCC/DPE), 91191 Gif-sur-Yvette, France \\ ${ }_{4}^{4}$ Commissariat à l'Énergie Atomique (CEA/DCC/DTE),26700 Pierrelatte, France \\ ${ }^{5}$ Office National d'Études et de Réalisations aérospatiales, BP 7292322 Châtillon cedex, France \\ ${ }^{6}$ Compagnie Industrielle des Lasers, BP 27, 91460 Marcoussis, France \\ ${ }^{7}$ Laboratoire Kastler-Brossel, École Normale Supérieure, 24 rue Lhomond, 75005 Paris, France \\ ${ }^{8}$ Laboratoire pour l'Utilisation des Lasers Intenses, École Polytechnique, 91128 Palaiseau, France \\ ${ }^{9}$ Physics Departement, National University of Ireland, Galway, Ireland
}

Adaptive optics at astronomical telescopes aims at correcting in real time the phase corrugations of incoming wavefronts caused by the turbulent atmosphere, as early proposed by Babcock. ${ }^{1}$ Measuring the phase errors requires a bright source located within the isoplanatic patch of the programme source. The probability that such a reference source exists is a function of the wavelength, of the required image quality (Strehl ratio), of the turbulence optical properties, and of the direction of the observation. It turns out that the sky coverage is disastrously low ${ }^{2}$ in particular in the visible wavelength range where, unfortunately, the gain in spatial resolution brought by adaptive optics is the largest. Foy and Labeyrie ${ }^{3}$ have proposed to overcome this difficulty by creating an articial point source in the sky in the direction of the observation relying on the backscattered light due to a laser beam. This laser guide star (hereafter referred to as LGS) can be bright enough to allow us to accurately measure the wavefront phase errors, except for two modes which are the piston (not relevant in this case) and the tilt. Pilkington ${ }^{4}$ has emphasized that the round trip time of the laser beam to the mesosphere, where the LGS is most often formed, is significantly shorter than the typical tilt coherence time; then the inverse-return-of-light principle causes deflections of the outgoing and the ingoing beams to cancel. The apparent direction of the LGS is independent of the tilt. Therefore the tilt cannot be measured.

Until now, the way to overcome this difficulty has been to use a natural guide star to sense the tilt. ${ }^{5-7}$ Although the tilt is sensed through the entire telescope pupil, one cannot use a faint source because $\approx 90 \%$ of the variance of the phase error is in the tilt. Therefore, correcting the tilt requires a higher accuracy of the measurements than for higher orders of the wavefront. ${ }^{8}$ Hence current adaptive optics devices coupled with a LGS face low sky coverage. ${ }^{9,2}$ Several methods have been proposed to get a partial sky coverage for the tilt. ${ }^{8,10,11}$ The only one providing us with a full sky coverage is the the polychromatic LGS $^{12,13}$ (hereafter referred to as PLGS).

We present here a progress report of the R\&D programme Étoile Laser Polychromatique et Optique Adaptative (ELP-OA) carried out in France to develop the PLGS concept. After a short recall of the principles of the PLGS, we will review the goal of ELP-OA and the steps to get over to bring it into play. We finally shortly described the effort in Europ to develop the LGS. 

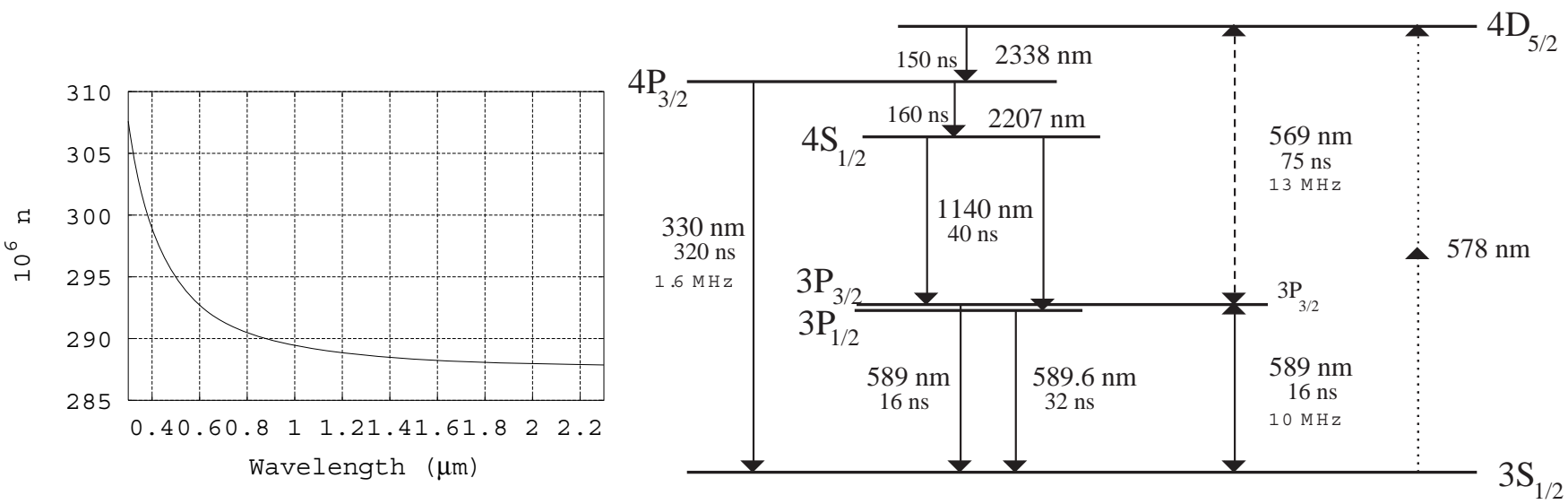

Figure 1. Variation of the refraction index of Figure 2. Diagram of the most significant energy levels of sodium. air with wavelength

Wavelengths (in $\mathrm{nm}$ ) and lifetime (in $\mathrm{ns}$ ) of the transitions are given, as well as the homogeneous widths (in $\mathrm{MHz}$ ) of the 589, 569, and 330 nm transitions.

\section{PRINCIPLE OF THE PLGS}

The concept of the PLGS relies on the chromatic properties of the refractive index of air, $n$. $n$ varies with the wavelength, quite abruptly in the ultraviolet range. Thus, a process which produces backscattered light at several different wavelength in the uppermost atmosphere, including ultraviolet lines, will cause the backscattered light to cross the atmosphere through different optical paths. Such a process can be stimulated by exciting mesospheric sodium atoms up to the $4 D_{5 / 2}$ energy level, from which the radiative decay down to the ground level produces a line spectrum spanning the wavelength interval from $330 \mathrm{~nm}$ to $2.3 \mu \mathrm{m}$, as shown in Fig. 2. A two-photon coherent absorption through the $3 P_{3 / 2}$ energy level and the 589 and $569 \mathrm{~nm}$ transition allows us to reach the $4 D_{5 / 2}$ energy level.

The monochromatic components of the polychromatic LGS appears slightly shifted in the sky: this is the differential tilt, $\delta \theta$. It is related to the tilt, $\theta$, through

$$
\delta \theta=\theta \times \delta n /(n-1),
$$

where $\delta n$ stands for the variation of $n$ over the wavelength baseline of the monochromatic components used for $\delta \theta$. More details about the conditions of this excitation of mesospheric sodium atoms are given in Foy et al.. ${ }^{13}$ Let us recall here the laser peak power of the saturation of the absorption in the two transitions : $I_{\text {sat }}=(h \nu) /(\sigma \tau)=$ $185 \mathrm{~W} \mathrm{~m}^{-2}$ and $475 \mathrm{~W} \mathrm{~m}^{-2}$ per spectral mode respectively for 589 and for $569 \mathrm{~nm}$, where $h$ is the Planck constant and $\nu, \sigma$ and $\tau$ are respectively the frequency, the cross section, and the lifetime of the transition, respectively.

The following topics have to be addressed in order to state that the concept of the PLGS is really able to provide tilt measurements

\section{- return flux at $330 \mathrm{~nm}$}

- telescope vibrations

- accuracy of the position measurements

- atomic physics model of the sodium atom

- budget link

\section{- R\&D of dedicated lasers}

The ELP-OA programme aims at addressing these topics in order to run a PLGS coupled with the adaptive optics device BOA developed by ONERA $^{14}$ at the $1.52 \mathrm{~m}$ telescope at Observatoire de Haute-Provence (OHP) and to observe long-exposure diffraction limited images without any natural reference source. In the following sections, we will describe results we have obtained by the end of the feasibility study of ELP-OA (December 1999). 


\section{RETURN FLUX AT 330 NM: THE PASS-2 EXPERIMENT}

Since the tilt is derived from a differential measurement, significantly more flux is required to determine the position of the monochromatic components of the PLGS than that of a natural guide star. This is particularly crucial for the $330 \mathrm{~nm}$ component, which has the maximum weight in the tilt determination because of the steep variation of $n$ around this wavelength. We had carried out a first experiment ${ }^{15,16}$ to measure the return flux at $330 \mathrm{~nm}$ at the Lawrence Livermore National Laboratory. It was the Polychromatic Artificial Star System experiment (hereafter referred to as PASS-1). The average laser power of the cv lasers used was $\approx 180 \mathrm{~W}$ for each of the beams locked on the two transitions. The return flux at $330 \mathrm{~nm}$ was $\approx 4.510^{5}$ photons $/ \mathrm{s} / \mathrm{m}^{2}$ for a pulse repetition rate of $4.3 \mathrm{kHz}$, a pulse FWHM of $50 \mathrm{~ns}$ and a modulation of $3 \mathrm{GHz}$ at $589 \mathrm{~nm}$ and $1 \mathrm{GHz}$ at $569 \mathrm{~nm}$. Increasing the repetition rate by a factor of 3 up to $12.9 \mathrm{kHz}$ had led to a decrease of the return flux by a factor of 2 . Poor weather conditions had prevented us from making measurements at lower powers which are required to fit the observations with atomic physics models. On the other hand, preliminary computations of sodium atom models predicted return fluxes significantly lower than those observed at the LLNL. Thus, we have carried out a second experimental campaign on the sky at the Commissariat à l'Énergie Atomique (hereafter referred to as CEA) at its Pierrelatte site. This was the PASS-2 experiment. The run lasted 6 weeks, in October and November of 1999. See Schöck et al. ${ }^{17}$ for an in-depth description and discussion of the PASS-2 experiment.

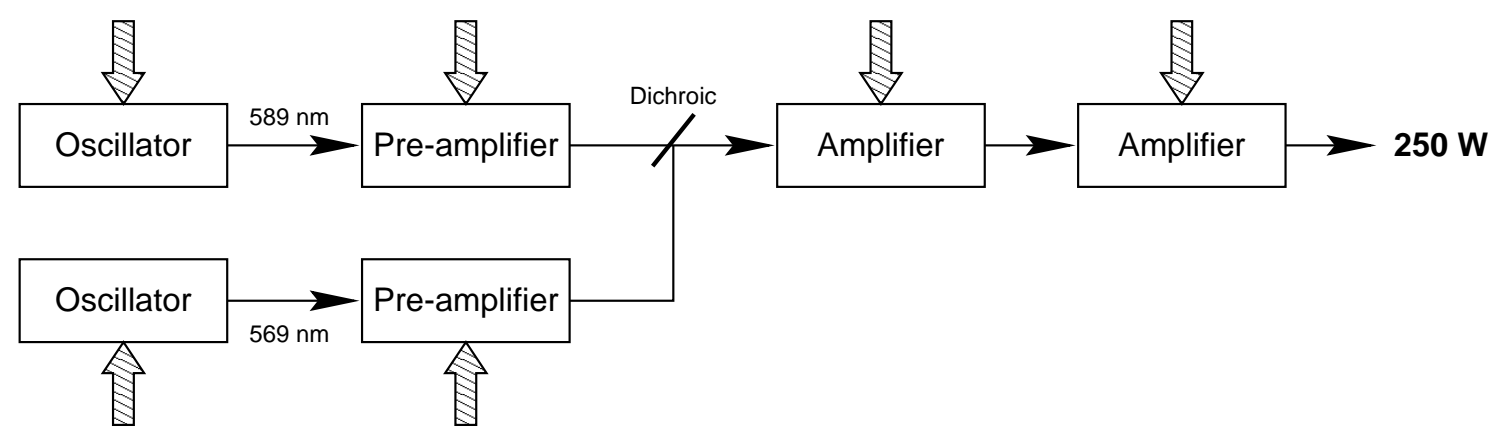

Figure 3. Schematic of the CEA laser chain used in the PASS-2 experiment.

The laser chain schematic is shown in Fig. 3. Dye lasers are pumped with $I C L 400 \mathrm{cv}$ lasers from CILAS. The parameters of the laser chain are given in Table 1.

Table 1. Principal characteristics of the laser configuration.

\begin{tabular}{|ll|}
\hline Total power in both colors & $10-100 \mathrm{~W}$ \\
Ratio of the powers in the two colors & $1: 1$ \\
Pulse repetition rate & 5 \\
Beam polarization & linear \\
Modulation & one modulator, $180 \mathrm{MHz}$ at $589 \mathrm{~nm}, 125 \mathrm{MHz}$ at $569 \mathrm{~nm}$, \\
& or 2 modulators, $180 \mathrm{MHz}$ and $300 \mathrm{MHz}$, at $589 \mathrm{~nm}$ \\
& broadened to $\approx 0.5 \mathrm{GHz}$ \\
Spectral characteristics & linewidth: $50 \mathrm{MHz}$ \\
& spectral jitter: $100 \mathrm{MHz}$ \\
Pulse shape & triangular \\
Pulse duration & $35 \mathrm{~ns}$ at FWHM, $70 \mathrm{~ns}$ at the base \\
Wave front quality & better than $1 \lambda$ \\
Beam diameter & $28 \times 34 \mathrm{~mm}^{2}$ \\
Precision of beam superposition & better than $10 \%$ of the diffraction limit \\
\hline
\end{tabular}

Two series of measurements of the return flux at $330 \mathrm{~nm}$ have been performed with two different oscillators. 
With the SILVA oscillators: We first used the CEA oscillators with either no modulation, or a single sine modulation (at $180 \mathrm{MHz}$ ), or a two-sine modulation (at 180 and $300 \mathrm{MHz}$ ) for the $589 \mathrm{~nm}$ beam and a single sine (at $125 \mathrm{MHz}$ ) for the $569 \mathrm{~nm}$ beam. In both the last cases, the modulation width was $\approx 0.5 \mathrm{GHz}$.

Return fluxes measured are shown in Fig. 4 . The return flux is $\approx 30,000$ photons $\mathrm{m}^{-2} \mathrm{~s}^{-1}$ at $\approx 50 \mathrm{~W}$ per beam. It is consistent with the measurements at the LLNL at $180 \mathrm{~W}$. Indeed since we are at saturation the return flux increases as the intensity, i.e. the return flux at the CEA is expected to be lower by a factor of 3.6. In addition, because of the narrower modulation bandwidth at the CEA, which spans only a part of the $\mathrm{F}=2$ component of the $589 \mathrm{~nm}$ transition, and taking into account the Rabi frequency, only a third of the velocity classes of the sodium atoms are excited in the $D_{2}$ transition. Then, the resulting theoretical ratio of the return flux at the LLNL versus that at the CEA is $\approx 11$. The sodium column density may also have been different during these two campaigns.

With the LSP oscillators: The modulation function of the laser spectral profile looks like a multi Dirac delta function, as long as the Rabi frequency does not fill the gap between the peaks (which would result in a poor efficiency). Then, it is mandatory that modulation functions of both beams are locked on the same radial velocity; if this is not the case, atoms excited by the $589 \mathrm{~nm}$ beam to the $3 P_{3 / 2}$ energy level may be not excited to the $4 D_{5 / 2}$ level by the $569 \mathrm{~nm}$ beam.

Spectral jitter of the lasers causes ramdomly variable shift of the beam spectral profile. This shift is different for the two beams. The oscillator of the SILVA chain has a spectral jitter width of $\approx 100 \mathrm{MHz}$. It is negligible with respect of the Rabi frequency at the highest peak power we have used $(\approx 350 \mathrm{MHz})$. But the spectral jitter is much larger than the homogeneous width (respectively 10 and $13 \mathrm{MHz}$ at 589 and $569 \mathrm{~nm}$ ). Then, at low values of the peak power, it would cause the excitation efficiency to drop significantly. Since we have to decrease the average laser power as much as possible in view of astronomical applications of the PLGS, the spectral jitter is a major concern. Therefore the LSP has developed an oscillator with a spectral jitter significantly lower than the homogeneous width of the transitions. It has been used during the PASS-2 experiment where it was amplified with the SILVA chain.

The jitter-free oscillator is built from a cw laser with a dye jet (599-21 Coherent), which is monomode and frequency locked. It is pumped by a YAG cw laser (VERDI/Coherent). Two such oscillators provide us with the two wavelengths at 589 and $569 \mathrm{~nm}$ with an accuracy $\lesssim 1 \mathrm{MHz}$. The two beams are then preamplified in two multi-pass devices with gains of up to 10,000 . The control of the wavelengths was done with a lambdameter (accuracy $\lesssim 50 \mathrm{MHz}$ ) and a sodium oven. The sodium oven allowed us to correlate the flux at $330 \mathrm{~nm}$ returned from the PLGS in the mesosphere with the laboratory measurements. This chain has been coupled with the CEA amplifiers to produce a laser beam at 589 and $569 \mathrm{~nm}$ launched into the mesosphere with a negligible spectral jitter. Because of the lack of time, the modulators and the beam stabilisation devices had not been transfered from the CEA oscillator to the LSP oscillator during this experiment. The rms fluctuation of the beam direction at one wavelength with respect to the other was $\approx 4$ arcsec; the beams were also superimposed quite poorly (in spite of a large seeing, $r_{0} \approx 5 \mathrm{~cm}$ ), which lowered the volume in the mesosphere where the two-photon excitation process could occur. The preliminary results given in Fig. 5 will be corrected for this bias in the final version of the data processing. Then, the effect of the spectral jitter on the efficiency of the process will be quantitatively estimated from the experimental measurements.

We ran two complementary experiments in parallel. The first one, conducted by the Galway team, had the purpose of measuring the sodium column density. Two instruments have been installed at the focus of a $30 \mathrm{~cm}$ telescope located $65 \mathrm{~m}$ from the launch telescope

- a LIDAR-like system (based on an APD and a boxcar), measuring the backscattered intensity as a function of time; it was synchronized with the laser pulses. Its purpose was in particular to correlate, if possible, time variations in the column density with sporadics.

- a CCD camera, with a lower spatial resolution than the APD.

Data acquired with these instruments will allow us to know the column density, which is an important parameter in the models. Data are currently being processed.

The second experiment is a Generalized Seeing Monitor ${ }^{18}$ (hereafter referred to as GSM) loaned by the Université de Nice-Sophia-Antipolis, of which the purpose was to provide measurements of Fried's parameter, $r_{0}$ simultaneously with the return flux measurements. Then, the laser spot area in the mesosphere can be determined and consequently the intensity, which is another critical parameter in the models. On average, we found $r_{0} \approx 4 \mathrm{~cm}$. The GSM has also provided us with a monitoring of the atmosphere transmission, allowing us to correct the measured return fluxes for variations in this parameter. The conditions either of seeing or of transmission were acceptable only during 4 nights. 


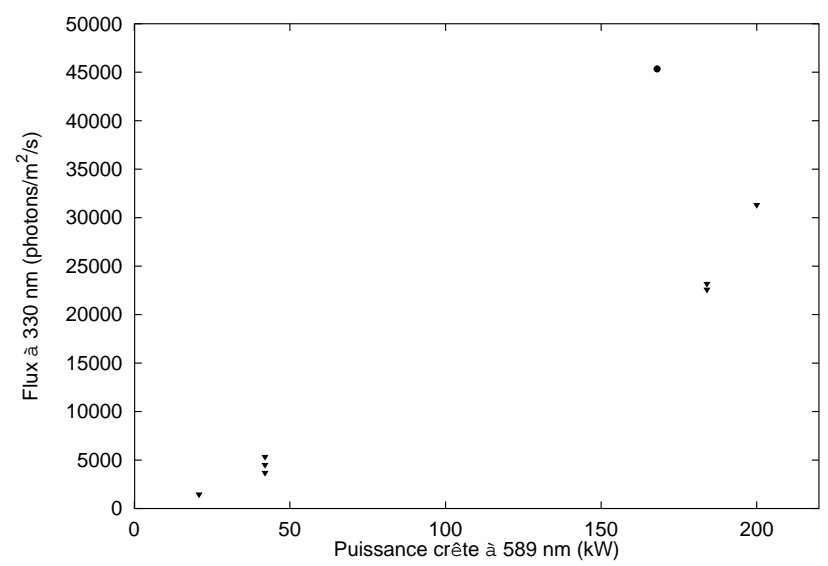

Figure 4. Return flux at $330 \mathrm{~nm}$ as a function of the laser peak power at $589 \mathrm{~nm}$. These measurements were taken using the CEA oscillator. The values shown apply to an atmospheric and instrument transmission of unity.

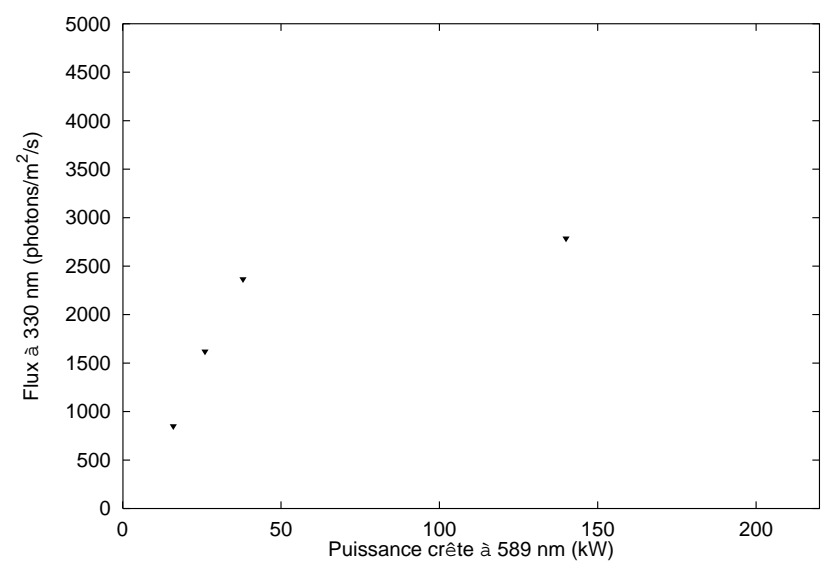

Figure 5. Same as Fig. 4 but using the the low-jitter oscillator of the LSP without frequency modulation.

\section{TELESCOPE VIBRATIONS: THE PENDULAR SEISMOMETER}

Since the concept of the PLGS relies on the wavefront propagation of the chromatic gradient of the refractive index of air, it does not permit us to correct for the mechanical causes of the image wandering such as telescope vibrations. Such vibrations are not negligible when compared with the telescope diffraction limit. ${ }^{19-21}$ At $H_{\alpha}$ and an $8 \mathrm{~m}$ telescope, an acceptable rms mechanical tilt of 7.0 marcsec degrades the Strehl ratio by a factor of 0.8 . Assuming monochromatic sinusoidal vibrations at $1 \mathrm{~Hz}$, the angular acceleration is $1.810^{-6} \mathrm{~s}^{-2}$, and the linear acceleration is $710^{-7} \mathrm{~g}\left(710^{-9} \mathrm{~g}\right.$ at $\left.0.1 \mathrm{~Hz}\right)$. Neither laser gyroscopes ${ }^{22,23}$ nor accelerometers have adequate performances.

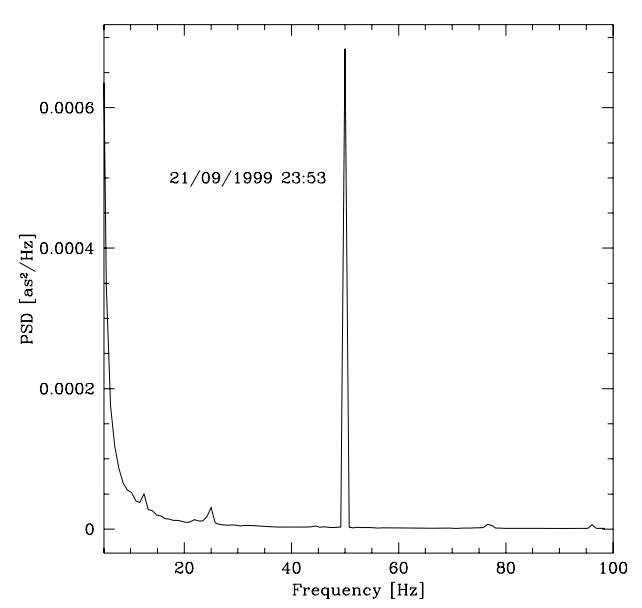

Figure 6. Vibrations at the OHP $1.52 \mathrm{~m}$ telescope, right ascension axis, telescope tracking on, dome open. The 6 mas amplitude peak at $50 \mathrm{~Hz}$ is a parasite. The peak amplitude at $76 \mathrm{~Hz}$ is $\lesssim 2$ marcsec.

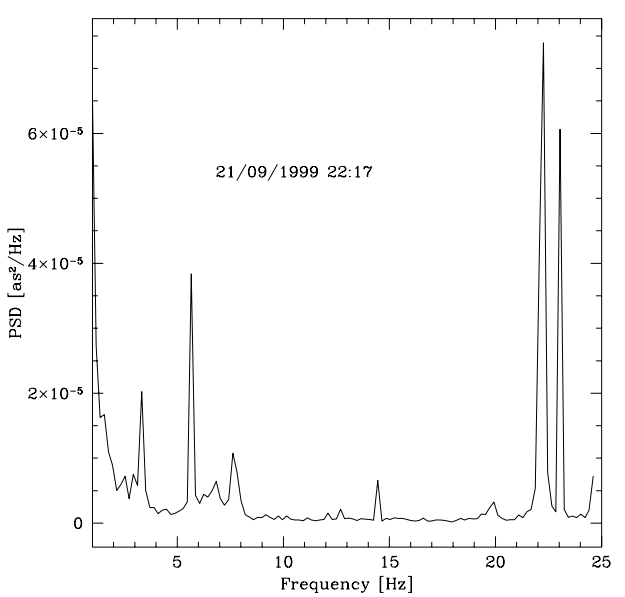

Figure 7. Vibrations at the Observatoire de HauteProvence $1.52 \mathrm{~m}$ telescope, declination axis, telescope tracking on, dome open.

Hence, a pendular seismometer ${ }^{24}$ has been conceived and built at Lyon Observatory to span the frequency range $0.1-\approx 30 \mathrm{~Hz}$. The principle is the following: a mass (a $1 \mathrm{~kg} 40 \mathrm{~cm}$ long arm of aluminum) is suspended at its center of gravity. Variations in the direction of the telescope axis are measured from the rotation of the arm with respect to the support of the seismometer, which is fixed to the telescope. Two such seismometers are required to measure 


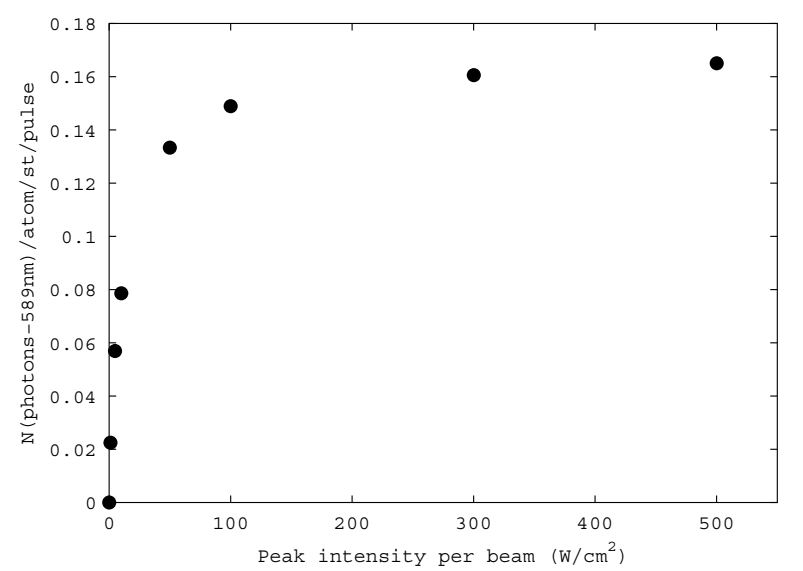

Figure 8. Predicted return flux at $589 \mathrm{~nm}$ versus the peak power per beam.

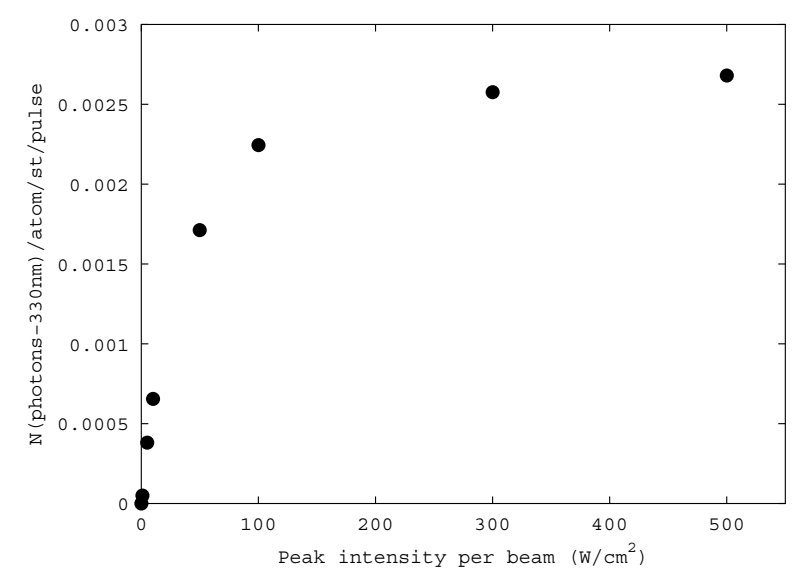

Figure 9. Predicted return flux at $330 \mathrm{~nm}$ versus the peak power per beam.

vibrations along the two telescopes axes. Two linear motors (LVDT) in opposite phase at each end of the arm servo its position with the tension applied providing the angular motion measurement.The measured rms noise of the pendular seismometer is 3 marcsec in the $0-25 \mathrm{~Hz}$ band: this prototype already matches the specifications for diffraction-limited imaging at 8-10 $\mathrm{m}$ class telescopes.

The goal of the next step of the ELP-OA project is to observe long exposure diffraction limited images at the OHP $1.52 \mathrm{~m}$ telescope. We have measured the vibration properties of this telescope with the pendular seismometer. Figure 6 and 7 show that they lie within the range of the usual tip-tilt mirror specifications.

\section{SODIUM ATOM MODELS: BACON CODE AND KINETICS EQUATION MODEL}

The code Bacon has been developped for the ELP-OA project. It aims at optimizing the photon flux (at $330 \mathrm{~nm}$ ) as a function of the laser excitation parameters (fluence, pulse duration, phase modulation parameters, etc.). A density matrix formalism has been used to solve the 48 equations of the two-step excitation process of the sodium atom considering the following energy levels: $S_{\frac{1}{2}}: F \in[1,2] ; 3 P_{\frac{3}{2}}: F \in[0,3] ; 4 D_{\frac{5}{2}}: F \in[1,4] ; 4 P_{\frac{3}{2}}: F \in[0 ; 3] ;$ and $4 S_{\frac{1}{2}}$ : $F \in[1,2]$; and for each $\left.F, M_{F} \in[-F, F]\right)$. The $4 D_{3 / 2}$ level has been neglected in the excitation ladder. The Doppler line profiles have been divided into several classes and the equations are solved for each class. The laser fields can be monomode or phase-modulated with different temporal shapes and different light polarizations. At the end, the populations of the considered levels and the number of photons emitted at 589, 569 and 330 nm are given as output. Results of this code have been compared, for validation, with those of Morris ${ }^{25}$ for a one-step excitation and with experiments. Predicted return fluxes at 330 and $589 \mathrm{~nm}$ are shown in Figs. 9 and 8 . At the average power $<P>=55$ $\mathrm{W}$ and with a single-sine modulation, the intensity at Pierrelatte was $\approx 9 \mathrm{~W} \mathrm{~cm}^{-2}$. Then, according to the figures the absorption at the sodium layer was saturated. Given an average laser power, a lower peak power and a higher pulse repetiton rate would therefore lead to higher return fluxes.

Because of the relatively large number of sub-levels, of the modulation function, and of the sampling of the line profiles $(\approx 100$ points per line), computation times for a single parameter configuration are large (several hours on a parallel computer). We have therefore developed a much faster code; it is based on the following kinetics equations:

$$
\begin{aligned}
\frac{\mathrm{d} N_{1}}{\mathrm{~d} t} & =-I_{1} \frac{N_{1}-N_{2}}{\tau_{2-1}}-\left[I_{1} I_{2}\left(N_{1}-N_{3}\right)\left(\frac{1}{\tau_{2-1}}+\frac{1}{\tau_{3-2}}\right)\right]+\frac{N_{2}}{\tau_{2-1}}+\frac{N_{4}}{\tau_{4-1}}+\frac{N_{6}}{\tau_{6-1}} \\
\frac{\mathrm{d} N_{2}}{\mathrm{~d} t} & =-I_{1} \frac{N_{1}-N_{2}}{\tau_{2-1}}-\frac{N_{2}}{\tau_{2-1}}-I_{2} \frac{N_{2}-N_{3}}{\tau_{3-2}}+\frac{N_{3}}{\tau_{3-1}}+\frac{N_{5}}{\tau_{5-2}} \\
\frac{\mathrm{d} N_{3}}{\mathrm{~d} t} & =-I_{2} \frac{N_{2}-N_{3}}{\tau_{3-2}}-\left[I_{1} I_{2}\left(N_{1}-N_{3}\right)\left(\frac{1}{\tau_{2-1}}+\frac{1}{\tau_{3-2}}\right)\right]-\frac{N_{3}}{\tau_{3-2}}-\frac{N_{3}}{\tau_{3-4}} \\
\frac{\mathrm{d} N_{4}}{\mathrm{~d} t} & =\frac{N_{3}}{\tau_{3-4}}-\frac{N_{4}}{\tau_{4-1}}-\frac{N_{4}}{\tau_{4-5}}
\end{aligned}
$$




$$
\begin{aligned}
\frac{\mathrm{d} N_{5}}{\mathrm{~d} t} & =\frac{N_{4}}{\tau_{4-5}}-\frac{N_{5}}{\tau_{5-2}}-\frac{N_{5}}{\tau_{5-6}} \\
\frac{\mathrm{d} N_{6}}{\mathrm{~d} t} & =\frac{N_{6}}{\tau_{5-6}}-\frac{N_{6}}{\tau_{6-1}}
\end{aligned}
$$

where the non-resonant terms are designed between the square brackets in Eqs. 2 and 4. We have calibrated these kinetics equations code by means of the Bacon code.

\section{ACCURACY OF MEASUREMENTS: THE MATILD EXPERIMENT}

The differential tilt is $\approx \frac{1}{20}$ of the tilt, from Eq. 1 . Thus, measuring the tilt at an accuracy of a fraction of the Airy disk from the differential tilt requires accuracies ranging around 1 mas. Our MaTilD experiment ${ }^{26}$ aims at testing whether this accuracy is achievable. A multi-wavelength (from 300 to $673 \mathrm{~nm}$ ) point source is generated and the beams are propagated horizontally over $\approx 100 \mathrm{~m}$. Data have first been simulated to evaluate the sources of noise and bias (photon noise, speckle noise, dark current, readout noise, slight overlapping of the monochromatic images). Figure 10 shows the correlation between the tilt and the tilt mesured from the differential tilt over a limited spectral basis, obtained from simulated data including all the identified sources of noise.

The gain in the rms error in the measurement of the image positions with respect to the center of gravity method is huge, according to these first estimates.

\begin{tabular}{llll}
\hline flux (counts) & $10^{4}$ & $10^{5}$ & $10^{6}$ \\
number of simulations & 100 & 100 & 500 \\
\hline \multicolumn{4}{c}{ RMS differential tilt error (pixels) } \\
centroid & 0.311 & 0.0397 & 0.0103 \\
$N_{\text {basis }}=3$ & 0.033 & 0.0062 & 0.0055 \\
$N_{\text {basis }}=4$ & 0.032 & 0.0061 & 0.0053 \\
$N_{\text {basis }}=5$ & 0.034 & 0.0057 & 0.0049 \\
$N_{\text {basis }}=6$ & 0.037 & 0.0054 & 0.0047 \\
$N_{\text {basis }}=7$ & 0.042 & 0.0056 & 0.0047 \\
$N_{\text {basis }}=8$ & 0.046 & 0.0047 & 0.0044 \\
$N_{\text {basis }}=9$ & 0.050 & 0.0052 & 0.0044 \\
\hline
\end{tabular}

Table 2. Precision for the differential tilt measured by different methods:the center of gravity method (first line named "centroid") and the fit of a phase screen model (following lines)

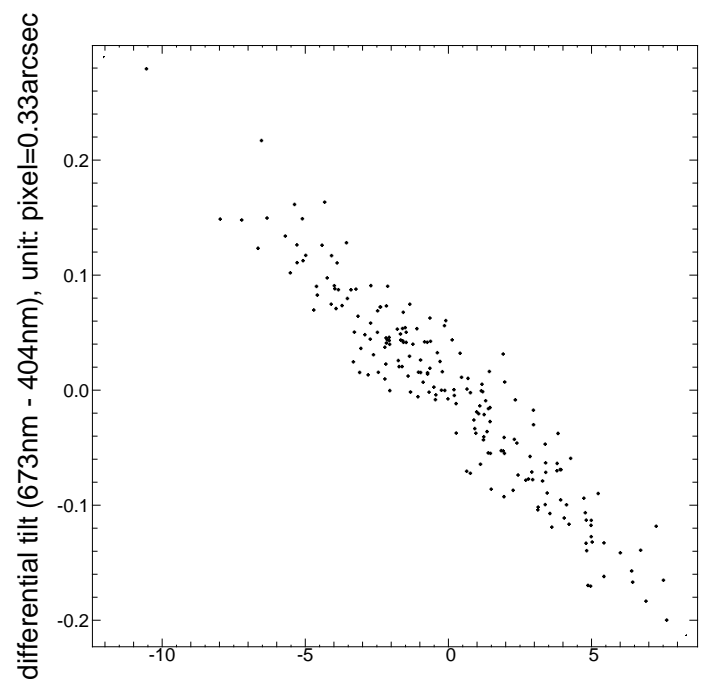

tilt at $673 \mathrm{~nm}$, unit: pixel=0.33arcsec

Figure 10. Correlation between differential tilt (673nm-404nm) and tilt (at 673nm), from 200 simulated images.

A phase mapping algorithm has been written to measure the differential tilt. The model $m_{\mathbf{x}}$ assumes that the data $d_{\mathbf{x}}$ are the superposition of the monochromatic components of the image of the polychromatic LGS

$$
m_{\mathbf{x}}=\sum_{\lambda} \alpha_{\lambda}\left|a_{\lambda, \mathbf{x}}\right|^{2}
$$

where $\alpha_{\lambda}$ is the intensity at the position $\mathbf{x}$ of the component at $\lambda$ and $\left|a_{\lambda, \mathbf{x}}\right|^{2}$ is the complex amplitude in the image plane. The game of the algorithm is to minimize the likelihood term $L(\mathbf{d} \mid \mathbf{m})$ of the data with respect to the model

$$
L(\mathbf{d} \mid \mathbf{m})=\sum_{\mathbf{x}} w_{\mathbf{x}}\left[d_{\mathbf{x}}-m_{\mathbf{x}}\right]^{2} \quad \text { with } \quad w_{\mathbf{x}}= \begin{cases}1 / \operatorname{Var}\left(d_{\mathbf{x}}\right) & \text { where data is available } \\ 0 & \text { elsewhere }\end{cases}
$$

of functions $b_{k, \lambda, \mathbf{u}}$ such as the tilt is expended only on the two first terms.

$$
\varphi_{\lambda, \mathbf{u}}=\beta_{\lambda} \sum_{k=1}^{N_{\text {basis }}} c_{k, \lambda} b_{k, \lambda, \mathbf{u}}
$$


where $\beta_{\lambda} \propto\left(n_{\lambda}-1\right) / \lambda$; therefore the coefficients of the expansion, $c_{k, \lambda}$, are identical but the two first ones, which have to be determined. The first estimates of the accuracy obtained with this algorithm in the determination of the position of the monochromatic components is very encouraging (see Table 2. The rms error is smaller than that obtained with the center of gravity algorithm by a factor 3 to 10 from $10^{6}$ to $10^{4}$ photons per image.

\section{BUDGET LINK}

The purpose of the end-to-end model ${ }^{17}$ of the PLGS process is to estimate the Strehl ratio of long exposure images as a function of the observation wavelength, $r_{0}$, the wavefront coherence time $\tau_{0}$, and the laser power. The Strehl ratio due to the tilt writes $\operatorname{as}^{27}$

$$
S_{t i l t}=\frac{1}{1+\frac{\pi^{2}}{2}\left(\sigma_{p h}^{2}+\sigma_{b w}^{2}\right)\left(\frac{D}{\lambda_{c}}\right)^{2}}
$$

where $\sigma_{p h}$ and $\sigma_{b w}$ stand for the photon noise standard deviation and for the bandwidth error respectively, where $\lambda_{c}$ is the observation wavelength, and where $D$ is the diameter of the telescope under consideration. Photon noise is expressed as

$$
\sigma_{p h}^{2}=\left(\frac{n-1}{\Delta n}\right)^{2} \frac{\sigma_{s p o t}^{2}}{\eta \Phi A_{r} t_{i}}
$$

where $\sigma_{\text {spot }}$ is the half-width at $1 / e$ of the LGS spot image on the detector, $\eta$ is the end-t0-end efficiency, $\Phi$ is the flux of photons at the ground (assuming no absorption and $\eta=1$ ), $A_{r}$ is the area of the receiving telescope, and $t_{i}$ is the integration time.

The temporal bandwidth variance is ${ }^{28}$

$$
\sigma_{b w}^{2}=\left(f_{T} / f_{3 d B}\right)^{2}(\lambda / D)^{2}
$$

where $f_{T}$ is the tilt coherence frequency, equivalent to the Greenwood frequency which accounts for all the modes

$$
f_{T}=0.331 D^{-1 / 6} \lambda_{c}^{-1} \sec ^{-1 / 2} \theta\left[\int \mathrm{d} h C_{n}^{2}(h) V(h)^{2}\right]^{-1 / 2} .
$$

Here, $\theta$ is the zenith angle, $C_{n}^{2}(h)$ is the turbulence structure constant as a function of altitude, $h$, and $V(h)$ is the wind velocity. $f_{3 d B}$ is the temporal $3 \mathrm{~dB}$ frequency of the correction system under the assumption that the transfer function of the system is given by

$$
H(f)=\frac{1}{1+i f / f_{3 d B}} .
$$

The integration time and the bandwidth of the system are then related by ${ }^{29}$

$$
f_{3 d B}=12^{1 / 4} \cdot\left(2 \pi t_{i}\right)^{-1} .
$$

Then we get

$$
\frac{S_{t i l t}=1}{1+a / t_{i}+b t_{i}^{2}}
$$

where $a \propto \lambda_{c}^{-2}$ and $b \propto f_{T}^{2}(0.5 \mu \mathrm{m}) \lambda_{c}^{-2}$. The integration time which lead to the maximum Strehl ratio is then given by

$$
t_{i}=\left(\frac{a}{2 b}\right)^{\frac{1}{3}}
$$

It is worthwhile noting that the best integration time is not dependent on the observation wavelength. Typical values range between 30 and $150 \mathrm{~ms}$.

Figures 11 and 12 show an example of the variation of the tilt-limited Strehl respectively versus the integration time and versus the observation wavelength for parameters typical of OHP. With these conservative parameters, a provisional estimate of the tilt-limited Strehl ratio is higher than $50 \%$ at $2.2 \mu \mathrm{m}$, and $\approx 8 \%$ at $550 \mathrm{~nm}$ in the visible. It assumes that the phase mapping method is used to measure the differential tilt. 


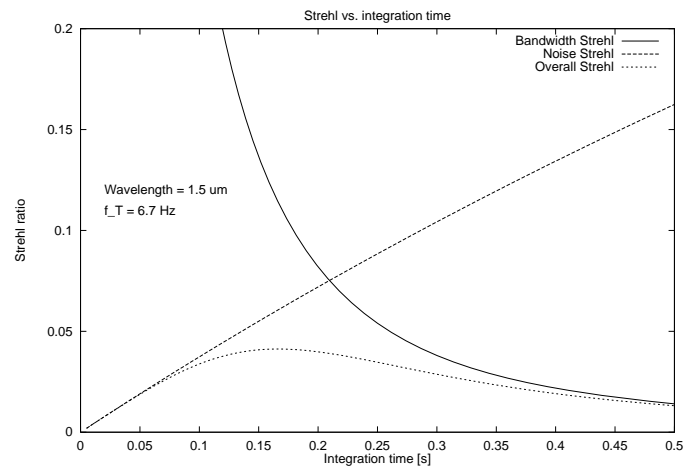

Figure 11. Strehl versus integration time for the case $f_{T}=6.7 \mathrm{~Hz}$ and $\lambda_{c}=1.5 \mu \mathrm{m}$ at Observatoire de Haute-Provence.

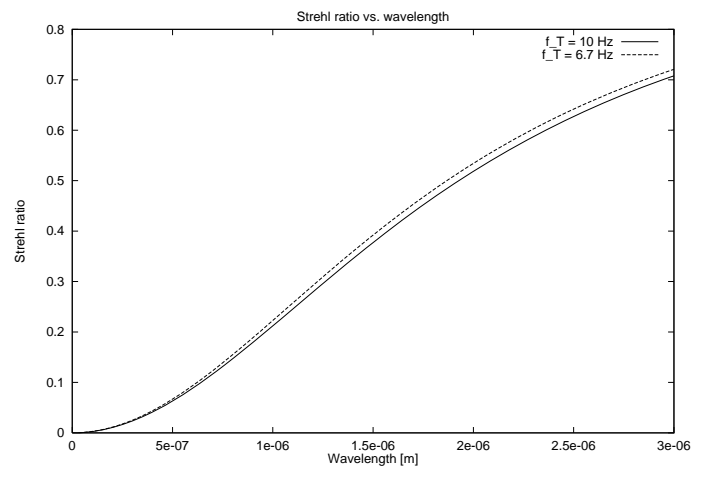

Figure 12. Strehl versus wavelength, $\lambda_{c}$, for the two cases of $f_{T}=10 \mathrm{~Hz}$ and $f_{T}=6.7 \mathrm{~Hz}$ and Observatoire de Haute-Provence typical parameters.

\section{THE LASER GUIDE STAR IN EUROPE}

The European Union, through its Training and Mobility Programme funds the Laser guide star for 8-meter class telescopes network wich allows European astronomers to coordinate their efforts in this field. Initially, this is a 4-year programme, which started in January 1997. We are applying for a one-year extension of the contract, which would thus end in December 2001. Participating institutes are CRAL/Observatoire de Lyon (leading), European Southern Observatory (international organisation, Germany), Imperial College (UK), Instituto de Astrofisica de Canarias (Spain), Max-Planck-Institute für Extraterrestrische (Germany), Osservatorio Astrophysica de Arcetri (Italie), and NUIG. This network aims at:

- studying the science which is expected with a laser guide star at a very large telescope; an analytical code of the Strehl ratio expected as a function of atmospheric parameters $\left(r_{0}\right.$, vertical profile of the turbulence and coherence time $\tau_{0}$ ), magnitude of the natural guide star or of the LGS, detector performances of the detector of the wavefront sensor, ...). Using either statistical properties of the distribution of stars in the sky (as a function of the galactic latitude) or crosscorrrelations of catalogues of stars with catalogues of objects of astrophysical interest, the sky coverage has been determined. It turns out to be particularly poor at visible wavelengths.

- providing a package of codes necessary to simulate the different steps or processes involved in the laser guide star concept. ${ }^{30}$ This package is named $C A O S$. It runs under the $I D L$ environment. All the package modules are cross-compatible. In particular the package include all the modules necessary to run an end-to-end simulation. It relies on a Monte-Carlo simulation. It includes

- a set of modules, each of them representing basic physical processes and basic components of an astronomical AO system.

- an application builder which permits to design simulation projects making use of the modular structure.

- a library of global utilities.

- templates with hints on writing new modules.

Many features can be modeled, as the effect of the Rayleigh cone on Shack-Hartmann sensing on a sodium LGS, modal filtering, turbulent atmosphere wind-shifting, multiple sources management, interferometry, etc.

- addressing the problems related to operational issues, such as safety (staff, airplanes, satellites), light pollution ${ }^{31}$ (detectors, on-site telescopes), laser technologies, infrastructure requirements and operational costs, tuning of the point spread function, ...

- progressing in the wavefront sensing:

- tilt measurement, as with the polychromatic guide star described in this paper, or with the perspective method $^{32,33}$ in which mobile auxiliary launch telescopes are used to sense the tilt of the beam backscattered in the mesosphere with respect to a natural guide star; 
- the cone effect $t^{3,34}$ which is due to the finite distance of the LGS whereas the programme objects are at infinity; the beams from the LGS which reach the edge of the primary mirror of the telescope do not cross the turbulent layers in the same location as the beams from the programme object, which results in a wrong measurement of the phase error and in a wrong correction applied to the deformable mirror. The 3-dimensional mapping of the turbulent layer allows us to reconstruct the total phase error along these beams. ${ }^{35,36}$ A very important byproduct is the widening of the corrected field which will be fully exploited with multiconjugate adaptive optics ${ }^{37,38}$;

- optimisation of the wavefront sensing for instance through the development of a new wavefront sensor which is the pyramid wavefront sensor ${ }^{39}$

- making use of the feed-back expected from the experiments carried on by the participants, such as the measurements of the seeing vertical distribution, ${ }^{40,41}$ the sodium column density in the mesosphere, ${ }^{42,43}$ the LGS - adaptative optics experiment ALFA ${ }^{44}$ and ELP-OA.

The ELP-OA programme take benefit from the R\&D programme of this network, in particular from the simulation package, from the ongoing experiments and sodium density monitoring, and from postdoc positions.

\section{CONCLUSIONS}

We have described the successful achievement of critical tasks of the feasibility study of the ELP-OA programme. This first phase of the ELP-OA has been completed at the end of 1999. The following forthcoming improvements in the budget link are expected

- good seeing site. Considering $r_{0} \approx 20 \mathrm{~cm}$ increases $f_{T}$ significantly and consequently the integration time for tilt measurements.

- larger launch telescope. Launching the beam with a larger diffraction-limited telescope leads to a smaller spot size in the mesosphere, and consequently to a smaller contribution of the photon noise to the Strehl degradation, assuming a fixed average laser power.

- cw lasers. Using cw lasers or lasers pulsed at high pulse repetition frequency would avoid to saturate the coherent two-photon absorption.

- phase screen mapping. Improving the accuracy of the differential tilt measurement is possible with the phase mapping algorithm. ${ }^{26}$

These improvements will lead either to improve the Strehl ratio for a given laser power, or reversely to decrease the laser power for a given Strehl ratio.

The next step of the ELP-OA programme is to set up an experiment at the $1.52 \mathrm{~m}$ telescope of Observatoire de Haute Provence where an adaptive optics system will be fed with a polychromatic laser guide star in order to obtain long-exposure diffraction-limited images without any natural guide star. The whole experiment will be optimized in order to decrease the average laser power if possible to $2 \times 25 \mathrm{~W}$. The oscillators, possibly reduced to a single device, will be pumped with a CuHBr laser, ${ }^{45}$ both developed at the LSP. The adaptive optics bench will be the BOA device developed at ONERA. ${ }^{14}$ The expected time for this second phase of the ELP-OA programme is three years.

\section{acknowledgements}

The ELP-OA is funded by the French Ministère de la Recherche (Contract number 98 B 0001) and Ministère de la Défense (Contract 95304 00012DSP), by the Région Rhône-Alpes (Contract L14840.00.00/97018030), by the CNRS (programmes ULTIMATECH and PNHRAA) and by the Université Claude Bernard Lyon-1 and Université Joseph Fourier at Grenoble. We thank L. Letoullec for having leaded the functional analysis of the programme. We are indebted to the staff of the mechanical and electronics shops of Lyon Observatory and of the mechanical shop of Laboratoire de Spectrométrie Physique at Grenoble. We thank P. Rabou at Laboratoire d'Astrophysique de Grenoble for optics computations. We also express our thanks to the staff of the CEA/DCC/DPE who welcame the PASS-2 experiment at Pierrelatte. All of these people played a major role in the success of the feasibility study of ELP-OA. We thanks C. Max at the LLNL and the Laser Clearing House and CNES for their help for satellite avoidance, and the French air traffic control for airplane safety. N.A., H.F., C.H. and M.S. benefit from a postdoc grant from the Laser Guide Star TMR European (contract number ERBFMRX-CT96-0094). 


\section{REFERENCES}

1. H. W. Babcock, "The possibility of compensating astronomical seeing," PASP 65, p. 229, 1953.

2. M. Le Louarn, R. Foy, N. Hubin, and M. Tallon, "Laser guide star for $3.6 \mathrm{~m}$ and $8 \mathrm{~m}$ telescopes: Performances and astrophysical implications," Mon. Not. R. astron. Soc 295, pp. 756-768, 1998.

3. R. Foy and A. Labeyrie, "Feasibility of adaptive telescope with laser probe," AA 152, pp. L29-32, 1985.

4. J. Pilkington, "Artificial guide stars for adaptive imaging," Nature 330, p. 116, 1987.

5. R. Davies, W. Hackenberg, T. Ott, A. Eckart, S. Rabien, S. Anders, S. Hippler, M. Kasper, P. Kalas, A. Quirrenbach, and A. Glindemann, "The science potential of ALFA: Adaptive optics with natural and laser guide stars," Astron. Astrophys. Suppl. Ser. 138, pp. 345-353, 1999.

6. S. S. Olivier, D. T. Gavel, H. W. Friedman, C. E. Max, J. R. An, K. Avicola, B. Bauman, J. M. Brase, E. Campbell, C. Carrano, J. Cooke, G. Freeze, E. Gates, V. Kanz, K. T, C, B. Macintosh, M. Newman, E. Pierce, K. Waltjen, and J. Watson, "Improved performances of the laser guide star adaptive optics at lick observatory," in Adaptive optics systems and technology, R. K. Tyson and R. Q. Fugate, eds., vol. 3762 of Proc. SPIE, 1999.

7. J. Sebag, C. d'Orgeville, M. Chun, J. Filhaber, J. Oschmann, F. Rigaut, and D. Simons, "Lick sodium laser guide star: performance during 1998 lgs observing campaign," in Adaptive optics systems and technology, R. K. Tyson and R. Q. Fugate, eds., vol. 3762 of Proc. SPIE, 1999.

8. F. Rigaut and E. Gendron, "Laser guide star in adaptive optics: the tilt determination problem," Astron. Astrophys. 261, pp. 677-684, 1992.

9. S. Olivier, C. Max, D. T. Gavel, and M. J. Brase, "Tip-tilt compensation - resolution limits for ground-based telescopes using laser guide star adaptive optics," Astrophys. J. 407, pp. 428-439, 1993.

10. R. Ragazzoni, "Robust tilt determination from laser guide star using a combination of different techniques," Astron. Astrophys. 319, pp. L9-L12, 1997.

11. M. Belenkii, S. J. Karis, J. M. B. II, and R. Q. Fugate, "Experimental validation of a technique to measure tilt from a laser guide star," Opt. Lett. 24, pp. 637-639, 1999.

12. R. Foy, Y. Boucher, B. Fleury, G. Grynberg, P. R. McCullough, A. Migus, and M. Tallon, "ATLAS status report and tilt sensing using multicolour laser reference star," in Progress in telescope and instrumentation technologies, U. M.-H., ed., no. 42 in ESO conferences, pp. 437-441, ESO, (Garching, Germany), 1992.

13. R. Foy, A. Migus, F. Biraben, G. Grynberg, P. R. McCullough, and M. Tallon, "The polychromatic artificial sodium star: a new concept for correcting the atmospheric tilt," AASup 111, pp. 569-578, 1995.

14. P.-Y. Madec, D. Rabaud, B. Fleury, J.-M. Conan, L.Rousset-Rouvière, F. Mendez, J. Montri, V. Michau, G. Rousset, and M. Séchaud, "Essais du banc d'optique adaptative ONERA à l'OHP," La Lettre de l'OHP 16, pp. 2-3, 1997.

15. R. Foy, M. Tallon, H. Friedman, A. Baranne, F. Biraben, G. Grynberg, M. L. Louarn, A. Petit, J.-M. Weulersse, A. Migus, and J.-P. Gex, "Polychromatic guide star," in High-Power Laser Ablation 1998, C. Phipps, ed., vol. 3343 of Proc. SPIE, pp. 194-204, 1998.

16. R. Foy, M. Tallon, E. Thiébaut, J. Vaillant, F.-C. Foy, D. Robert, H. Friedman, F. Biraben, G. Grynberg, J.-P. Gex, A. Mens, A. Migus, J.-M. Weulersse, and D. J. Butler, "Observation of a polychromatic laser guide star," J. Opt. Soc. Am. A submitted, 2000.

17. M. Schöck, R. Foy, J.-P. Pique, P. Chevrou, N. Ageorges, A. Petit, V. Bellanger, H. Fews, F.-C. Foy, C. Högemann, M. Laubscher, O. Peillet, P. Segonds, M. Tallon, and J.-M. Weulersse, "PASS-2: Quantitative photometric measurements of the polychromatic laser guide star," in Adaptive Optical Systems Technology, vol. 4007 of Proc. SPIE, 2000.

18. J. Vernin and M. Azouit, "Traitement d'image adapté au speckle atmosphérique. II. Analyse multidimensionnelle appliquée au diagnostic à distance de la turbulence," J. Optics (Paris) 14, pp. 131-142, 1983.

19. E. Thiébaut, Y. Balega, I. Balega, I. Belkine, J. Bouvier, R. Foy, A. Blazit, and D. Bonneau, "Orbital motion of DF Tauri from speckle interferometry," Astron. Astrophys. Lett. 304, pp. L17-L20, 1995.

20. E. J. Kibblewhite and M. R. Chun, "Design of tip-tilt and adaptive optics servos using measured angle-ofarrival and phase power spectra," in Adaptive Optical System Technologies, D. Bonaccini and R. K. Tyson, eds., vol. 3353 of Proc. SPIE, pp. 522-530, 1998.

21. S. Altarac, P. Berlioz-Arthaud, E. Thiébaut, and R. Foy, "Effect of telescope vibrations upon high angular resolution imaging," in preparation, 2000. 
22. W. W. Chow, J. Gea-Banacloche, L. M. Pedrotti, V. E. Sanders, W. Schleich, and M. O. Scully, "The ring laser gyro," Rev. Modern Phys. 57, pp. 61-104, 1985.

23. H. Dahlman, B. Huber, and W. Schröder, "Optical gyro encoder tested on the NTT," The Messenger 74, pp. 5-6, 1993.

24. A. A. Tokovinin, "Pendular seismometer for compensating telescope vibrations," MNRAS in press, 2000.

25. J. Morris, "Efficient excitation of a mesospheric sodium laser guide starby intermediate-duration pulses," $J$. Opt. Soc. Am. A 11, pp. 832-845, 1994.

26. J. Vaillant, É. Thiébaut, and M. Tallon, "ELPOA: Data processing of chromatic differences of the tilt measured with a polychromatic laser guide star," in Adaptive Optical Systems Technology, vol. 4007 of Proc. SPIE, 2000.

27. D. G. Sandler, S. Stahl, J. Angel, M. Lloyd-Hart, and D. McCarthy, "Adaptive optics for diffraction-limited infrared imaging with 8-m telescopes," J. Opt. Soc. Am. A 11, pp. 925-945, 1994.

28. G. A. Tyler, "Bandwidth considerations for tracking through turbulence with 8-m telescopes," J. Opt. Soc. Am. A 11, pp. 358-367, 1994.

29. R. R. Parenti and R. J. Sasiela, "Laser-guide-star systems for astronomical applications," J. Opt. Soc. Am. A 11, pp. 288-309, 1994.

30. M. Carbillet, bruno Femenia, F. Delplancke, S. Esposito, L. Fini, A. Riccardi, É. Viard, N. Hubin, and F. Rigaut, "La3os2: a software package for adaptive optics systems," in Adaptive optics systems and technology, R. K. Tyson and R. Q. Fugate, eds., vol. 3762 of Proc. SPIE, pp. 378-389, 1999.

31. N. Ageorges, N. Hubin, and F. Delplancke, "Laser guide star: monitoring and light pollution," in Adaptive Optical Systems Technology, vol. 4007 of Proc. SPIE, 2000.

32. R. Ragazzoni, S. Esposito, and E. Marchetti, "Auxiliary telescopes for the absolute tip-tilt determination of a laser guide star," Mon. Not. R. astron. Soc 276, p. L76, 1995.

33. S. Esposito, R. Ragazzoni, A. Riccardi, C. O.'Sullivan, M. Redfern, and R. Davies, "Absolute tilt from a laser guide star: a first experiment," Exp. Astron. in press, 2000.

34. M. Tallon and R. Foy, "Adaptive optics with laser probe: isoplanatism and cone effect," Astron. Astrophys. 235, pp. 549-557, 1990.

35. M. L. Louarn and M. Tallon, "Solving the cone effect: 3d mapping of turbulence," in Adaptive Optical Systems Technology, vol. 4007 of Proc. SPIE, 2000.

36. M. L. Louarn, J. C. Dainty, R. Foy, C. Paterson, and M. Tallon, "3d mapping of turbulence: laboratory experiment," in Adaptive Optical Systems Technology, vol. 4007 of Proc. SPIE, 2000.

37. F. Rigaut, "Performance and fundamental limitations of multiconjugate ao," in Adaptive Optical Systems Technology, vol. 4007 of Proc. SPIE, 2000.

38. N. Hubin, M. L. Louarn, M. Sarazin, and A. A. Tokovinin, "New challenges for adaptive optics: the owl 100m telescope," in Adaptive Optical Systems Technology, vol. 4007 of Proc. SPIE, 2000.

39. R. Ragazzoni, S. Esposito, M. Ghigo, A. Baruffolo, G. Crimi, A. Ghedina, E. Marchetti, and A. Riccardi, "Testing the pyramid wavefront sensor on the sky," in Adaptive Optical Systems Technology, vol. 4007 of Proc. SPIE, 2000.

40. J. Vernin and C. Muũnoz-Tunón, "Measuring astronomical seeing: the DA/IAC DIMM," Pub. Astron. Soc. Pacific 107, pp. 265-272, 1995.

41. N. J. Wooder, F. Rigal, C. Paterson, and J. C. Dainty, "Atmospheric turbulence profile measurement using scidar," in Adaptive Optical Systems Technology, vol. 4007 of Proc. SPIE, 2000.

42. S. Chueca and J. J. Fuensalida, "Monitoring of atmospheric sodium: new method to get the telluric spectrum and influence of the water lines in the performances of lgs," in Adaptive Optical Systems Technology, vol. 4007 of Proc. SPIE, 2000.

43. L. Michaille, T. A. Canas, J. C. Dainty, T. S. Gregory, J. Mawwell, J. C. Quartel, F. Reavell, and N. J. Wooder, "Monitoring the variation of mesospheric sodium density at la palma," in Adaptive Optical Systems Technology, vol. 4007 of Proc. SPIE, 2000.

44. A. Quirrenbach, W. Hackenberg, H.-C. Holstenberg, and N. Wilnhammer, "The ALFA dye laser system," in Laser technology for laser guide star Adaptive optics, N. Hubin, ed., no. 55 in ESO Workshops, pp. 126-131, ESO, (Garching), 1997.

45. P. Coutance and J.-P. Pique, "Radial and time resolved measurement of cuprous bromide concentration in a $\mathrm{Cu} / \mathrm{HBr}$ laser," IEEE J. Quantum Electron., 1998. 